\title{
Validation and Reliability of the Gujarati Version of the Physical Activity Readiness Questionnaire for Everyone (PAR-Q+)
}

\author{
Mansi Shingala ${ }^{1}$, Yagna Shukla ${ }^{2}$ \\ ${ }^{1}$ MPT, PhD Scholar- Gujarat University; Tutor cum Physiotherapist at Govt. Physiotherapy College, Jamnagar. \\ ${ }^{2} \mathrm{MPT}, \mathrm{PhD}$; Senior Lecturer at Govt. Spine Institute and Govt. Physiotherapy College, Ahmedabad.
}

Corresponding Author: Mansi Shingala

\begin{abstract}
Background: PAR-Q+ is self reported questionnaire and majorly used as a part of screening and to rule out the risk criteria before getting engaged in any physical activity program. As it is the self reported screening tool, it is necessary to translate the English questionnaire into the regional language.

Method: PAR-Q+ was translated into Gujarati by using forward-backward-forward translation method. Reliability of the translated final Gujarati version was assessed on 30 participants with different age group.

Result: There is an excellent internal consistency (Cronbach' alpha $=1 ; \mathrm{p}<0.05$ ) and almost perfect test retest reliability $(\mathrm{K}=1 ; \mathrm{p}<0.05)$ found for PAR- $\mathrm{Q}+$ Gujarati version. Spearman's correlation coefficient $(\rho=1)$ suggested Significant correlation between original English and translated Gujarati scales.
\end{abstract}

Conclusion: This Gujarati version of PAR-Q+ can help many of the Gujarati speaking people to screen themselves before participating any physical activity program.

Key Words: Gujarati PAR-Q+, translation of PAR-Q+, validity and reliability of Physical activity readiness questionnaire.

\section{INTRODUCTION}

Participation in regular physical activity can give many health benefits as well as it can help in prevention or delaying of many health related and life style disorders. Before getting engage in any of the physical activity program it is important to get the medical clearance to prevent any untoward incidences associated with physical activity participation. Physical Activity Readiness Questionnaire (PAR$\mathrm{Q}+$ ) is one such screening tool that can help the individual to rule out any risk factors before taking part in any kind of exercise session. PAR-Q + is evidence based questionnaire for pre screening and risk stratification for all the age groups and also for those who are having any associated medical conditions. This questionnaire include 7 general health questions and if anyone answers 'yes' to any of the question then they need to complete 10 follow up questions about the medical conditions along with the sub questions. From total 48 Questions; General health Questions include:

1) Has your doctor ever said that you have heart condition or high blood pressure?

2) Do you feel pain in your chest at rest, during your daily activities of living or when you do physical activity?

3) Do you lose balance because of dizziness or have you lost consciousness in last 12 months? 
4) Have you ever been diagnosed with another chronic medical condition (other than heart disease or high Blood pressure)?

5) Are you currently taking prescribed medications for a chronic medical condition?

6) Do you currently have (or have had within past 12 months) a bone, joint, or soft tissue (muscle, ligament or tendon) problem that could be made worse by becoming more physically active?

7) Has your doctor ever said that you should only do medically supervised physical activity?

Many a times it is found that benefits of regular physical activity are always more compared to the risk associated with physical activity. Considering that many people can take help of this self screening tool and can participate in physical activity program without the consultation with the physician. PAR-Q+ is widely accepted pre-screening tool and it is being used worldwide as pre-participatory requirement for any physical activity $\operatorname{program}^{1,2,3}$.

In that case, it is necessary to get the reliable translated version of PAR-Q+ for helping large number of people, only speaking their native/regional language, with the self screening tool. With the same motive, this study was conducted to translate the PAR-Q+ 2020 version into Gujarati and evaluate the reliability of the Gujarati version of PAR-Q+.

\section{METHOD}

The translation process was conducted after receiving permission from the original authors of PAR-Q+ through mail. $^{4}$

Translation of PAR-Q+ into Gujarati was done by using forward-backwardforward translation method (Beaton Guidelines, 2000). For Face and Content Validity; translation process started with the two independent translators, who were bilingual and have sound knowledge of both the languages, translated the questionnaire into Gujarati ( $\mathrm{T} 1$ and $\mathrm{T} 2$ ). Then the researchers have produced a combined Gujarati version (T12) of PAR-Q+ from the two independent Gujarati translations. Then this version (T12) was given to two different translators for back translation (BT1 and BT2). The back translated versions were then compared with the original English PAR Q+. As there was good match of these versions with the original one, the Combined Gujarati version T12 was then given to the expert panel consisted of 7 members from medical as well as non medical background and having minimum of 10 years of experience in their respective fields (Medicine, Language, Physiology and Physiotherapy). Agreement with the questions by at least $80 \%$ can be considered for group consensus method. Each of the panel members was personally contacted by primary author for the expert opinion. Assessment criteria were kept as content and meaning of question, formation and easiness of administration. Each question was scored as accepted, rejected or accepted with modification along with the remarks. All the members accepted the translated version with $>80 \%$ agreement for all the 48 questions. Then the final translated Gujarati Questionnaire and original English Questionnaire were given to the 30 individual (Target population) for Construct validity measurement. Inclusion criteria were kept as age $>=20$ years, both male and female and having knowledge of both Gujarati and English language. Individuals who were not willing to participate were excluded. Both Original English and translated Gujarati Questionnaires were given in random order (odd numbers were given English version first and Even numbers were given Gujarati version first to complete) one day apart. Then the answers from Gujarati Questionnaire were compared with the answers of original English Questionnaire. For Test retest reliability, translated Gujarati Questionnaire was once again given to the same participants one week apart for test retest reliability measurement ${ }^{5-9}$. 


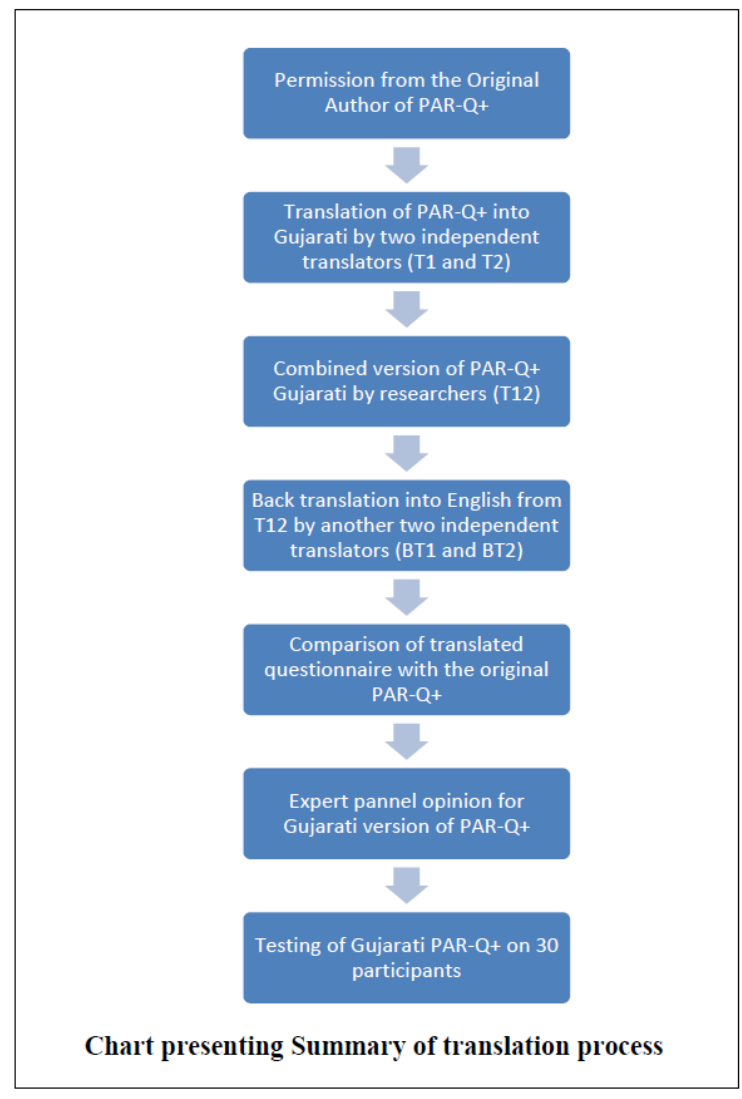

\section{Statistical Analysis:}

Statistical analysis was done by using SPSS (Statistical package for the social sciences) version 20. Kappa statistics was used to assess test retest reliability at 95\% confidence interval. Kappa values are interpreted as ${ }^{3}$ :

$<0=$ No agreement

$0-0.19=$ Poor agreement

$0.20-0.39=$ Fair agreement

$0.40-0.59=$ Moderate agreement

0.60-0.79 $=$ Substantial agreement

0.80-1.00 = Almost perfect agreement

Cronbach's alpha $(\alpha)$ was assessed for internal consistency with $\mathrm{p}<0.05$. Value of $\alpha$ ranges from $0-1$; higher value indicates greater internal consistency.

Spearman's correlation coefficient ( $\rho)$ was calculated for correlation between English and Gujarati version of PAR-Q+.

\section{RESULT}

Total 30 participants with mean age of $41.40 \pm 16.34$ years completed the Questionnaire. For analysis of answers given by participants in form of 'yes' and 'no' were coded as ' 1 ' and '2' respectively. When analysed, results showed an excellent internal consistency (Cronbach'alpha $=1$; $\mathrm{p}<0.05)$ and almost perfect test retest reliability $(\mathrm{K}=1 ; \mathrm{p}<0.05)$ for all the questions of PAR- Q+ Gujarati version. Spearman's correlation coefficient $(\rho)$ was calculated for correlation between English and Gujarati version of PAR-Q+ and $\rho=1$ suggested Significant correlation between both the scales.

\section{DISCUSSION}

PAR-Q+ has been translated into many regional languages. As per our knowledge, this is the first study done on translation of PAR-Q+ into Gujarati and validation of Gujarati version of PAR-Q+. Results showed excellent internal consistency and almost perfect test retest reliability for Gujarati PAR-Q+ along with significant correlation between English and Gujarati version of PAR-Q+. This suggests that translated version of PAR-Q+ is easily and fully understandable Gujarati Questionnaire for Gujarati speaking population. The Gujarati PAR-Q+ can help the people who want to join the physical activity programme with self screening method. Translated version can engage more Gujarati speaking people to start physical activity and can prevent them from inactivity life style disorders. This self administered and cost effective Questionnaire can be completed in very few time and can save the time from getting medical clearance by physician for participation in physical activity ${ }^{3,10}$.

\section{CONCLUSION}

Gujarati version of PAR Q+ is valid and reliable tool that can help many of the Gujarati speaking people to screen themselves before participating any physical activity program.

\section{ACKNOLEDGEMENT}

Authors would like to acknowledge the Original authors of PAR-Q+ for providing permission for the translation 
process. We would like to acknowledge all the members of expert panel and all the participants who have helped in the Gujarati translation and validation process of PAR$\mathrm{Q}+$.

\section{Conflict of Interest: None}

\section{Source of Funding: None}

\section{REFERENCES}

1. Warburton D E, Bredin S S, Jamnik V K and Gledhill N. Validation of the PAR-Q+ and ePARmed-X+. The health and Fitness journal of Canada, 2011; 4(2):38-46.

2. Warburton D E, Jamnik V K, Bredin S S, Shephard R J and Gledhill N. The 2020 Physical Activity Readiness Questionnaire for Everyone (PAR-Q+) and electronic Physical Activity Readiness medical examination (ePARmed-X+):2020 PARQ+. The health \&Amp; Fitness Journal of Canada,2019;12(4):58-61.

3. Schwartz J. et al. Cross cultural translation, adaptation and reliability evaluation of the Spanish version of the physical Activity Readiness Questionnaire for Everyone (PAR-Q+). Health and fitness journal of Canada, 2019; 12(4):3-14.

4. Darren Warburton. "Re: Regarding translation of PAR-Q+." 2020. Email.

5. Beaton E D and Guillemin F. Guidelines for the process of Cross-Cultural adaptation for
self-Report Measures. SPINE, 2000; 25(24): 3186-3191.

6. Rathod S R, Vyas N and Sorani D M. Validation of Gujarati version of International Physical Activity Questionnaire. Int $\mathbf{J}$ of Health Sci Res, 2018; 8(1): 122-128.

7. Koo $\mathrm{T} \mathrm{K}$ and Li M Y. A Guideline of selecting and reporting Intraclass correlation Coefficients for Reliability Research. Journal of Chiropractic medicine. 2016; 15 : 155-163.

8. Cha E S, Kim K H and Erlen J A. Translation of scales in Cross-cultural research: issues and techniques. Journal of Advanced Nursing, 2007; 58(4): 386-395.

9. Taherdoost H. Validity and reliability of the research Instrument; How to Test the Validation of a Questionnaire/Survey in a Research. International Journal of Academic Research in Management, 2016; 5(3):28-36.

10. Ertekin Y H. Turkish adaptation of the physical Activity Readiness Questionnaire for Everyone (PAR-Q+). Farm Pract palliate Care, 2018; 3(1):52-64.

How to cite this article: Shingala M, Shukla Y. Validation and reliability of the Gujarati version of the physical activity readiness questionnaire for everyone (PAR-Q+). Int J Health Sci Res. 2021; 11(6): 290-293. DOI: https://doi.org/10. 52403/ijhsr.20210642 